\title{
Indicadores sociales para una planificación educativa en la comunidad de Castilla-La Mancha
}

\author{
Octavio Uña Juárez \\ José María Bleda García \\ Gema Jiménez Tostón
}

\section{Resumen}

El texto resume los principales aspectos de la evolución de la población, movimientos migratorios y otras variables sociodemográficas útiles para la planificación educativa en La Mancha.

Palabras clave: indicadores sociales, planificación educativa, demografía regional.

Abstract. Social indicators for a educational planning in the Castilla-La Mancha region

The text sumarizes the main aspects of the population evolution of the Spanish region Castilla-La Mancha in order to make education planning feasible.

Key words: social indicators, educational planning, regional demography.

\begin{aligned} & \multicolumn{2}{c}{ Sumario } \\ & 1. Introducción 5. Formación \\ & 2. Aspectos demográficos 6. Población activa e inactiva \\ & 3. Hogares y viviendas 7. Conclusiones \\ & 4. Movimientos migratorios Bibliografía \end{aligned}

\section{Introducción}

Una planificación educativa ha de tener en cuenta determinados indicadores sociales que le sirvan para llegar a la realidad social de manera más ajustada y válida. Consideramos que las aportaciones sociológicas que se van a exponer a continuación pueden ser útiles a la hora de elaborar un futuro modelo educativo para la comunidad de Castilla-La Mancha. Entre los aspectos socioeconómicos que habría que analizar hemos destacado los referidos a la población, los hogares y viviendas, los movimientos migratorios y la población activa e inactiva, ya que tales factores pueden ayudar a una mayor comprensión de la realidad social y de su correlación con el sistema educativo. 
Las fuentes de datos que hemos utilizado son de muy variada procedencia. Se han utilizado principalmente el Censo de Población y Viviendas de 1991 y la Encuesta Sociodemográfica de 1991. En estas fuentes hemos realizado una explotación rigurosa de los datos que podían facilitar información interrelacionada con el sistema educativo. Además, hemos consultado otras fuentes secundarias como el Informe sociológico sobre la situación social en España (FOESSA, 1994) y el Informe anual sobre la realidad social española (CECS, 1994), ya que dichos informes contienen diversos apartados dedicados a la educación, así como los resultados de la Estadística de la Enseñanza en España para el curso 1992/93 (MEC, 1995).

\section{Aspectos demográficos}

\subsection{El poblamiento}

La región de Castilla-La Mancha está compuesta por las provincias de Albacete, Ciudad Real, Cuenca, Guadalajara y Toledo, con una extensión de $79.230 \mathrm{~km}^{2}$ y una población de derecho, en el año 1991, de 1.658.445 habitantes, lo que supone una densidad de 20,93 habitantes por $\mathrm{km}^{2}$; densidad que si es observada a nivel provincial aún es más reducida, pues en Guadalajara y Cuenca es de $11,94 \mathrm{~h} / \mathrm{km}^{2}$ y de $12,03 \mathrm{~h} / \mathrm{km}^{2}$ respectivamente. Es un espacio muy despoblado, ya que sus efectivos son un $4,26 \%$ con respecto al total nacional, si bien su superficie abarca el 16\% de la española. Y si comparamos la densidad de la Región con la de España, que en el año 1993 es de $78 \mathrm{~h} / \mathrm{km}^{2}$, y con la de Europa de los 12 , que es de $147 \mathrm{~h} / \mathrm{km}^{2}$, podemos concluir que es una región muy despoblada.

En cuanto al tipo de poblamiento, una de sus principales características demográficas es el desequilibrio territorial. Las poblaciones se sitúan en las provincias más meridionales (Toledo, Ciudad Real y Albacete) y se concentran en los núcleos de mayor tamaño. El espacio mayor está dominado por pequeños municipios, con una población regresiva, lo que supone la existencia de amplias áreas subpobladas con tendencia a la desaparición. En las provincias de Cuenca y Guadalajara, su población se concentra en las capitales provinciales; en las otras provincias (Âlbacete tiene una situación intermedia) existe un número de municipios urbanos o semiurbanos mejor dotados para organizar su espacio circundante.

$\mathrm{Al}$ analizar la población a nivel municipal se observa un gran número de municipios, 915, si bien la mayoría de ellos se sitúa en las provincias más despobladas: Guadalajara con 287 municipios y Cuenca con 238. Buena parte de los municipios (495) está habitada por menos de 500 habitantes, determinando débiles concentraciones, incluso en sus ciudades. La población de la región se agrupa preferentemente en núcleos de población, ya que sólo un mínimo porcentaje se encuentra en diseminado. En la zona urbana (a partir de 10.001 habitantes) el porcentaje en núcleos de población es del 99,32\% en la zona intermedia (entre 2.001 y 10.000 habitantes) del 99,45\%, en la zona rural (menos de 2.000 habitantes) del $97,80 \%$. Estas cifras son casi iguales a las 
que corresponden a las provincias, si bien hay una pequeña diferencia en la zona rural: si la media de la región en diseminado es del 2,20\%, en Cuenca es del $0,60 \%$, en Guadalajara es un $1,75 \%$, en Toledo un 2,18\%, y en Albacete y Ciudad Real es un 3,77\% y 3,16\% respectivamente.

\subsection{La evolución de la población}

En la evolución de la población castellano-manchega a lo largo del siglo XX observamos tres etapas: de 1900 a 1950 , donde hay un crecimiento de la población del $46,5 \%$, pasándose de una población de 1.386 .143 habitantes a 2.030 .598 habitantes; de 1950 a 1981 , caracterizada por una despoblación masiva, se pierden 402.593 habitantes; y de 1981 a 1991, definida por una cierta recuperación de la población.

En la primera etapa (1900-1950) la evolución tiene que ver con la situación económica tanto nacional como regional, no se había iniciado aún con la revolución industrial, éramos un país meramente agrario; la región de Castilla-La Mancha estaba «inmersa en una economía apoyada por el sector primario, incluso más floreciente que en otras zonas españolas, lo cual contribuía a mantener unos niveles demográficos» ${ }^{1}$. A partir de 1950 se inicia un proceso de despoblamiento, que se acentúa entre 1960 y 1970 , la población disminuye en un $20 \%$ a causa de la emigración a regiones ya industrializadas. A partir de 1981 se observa un leve aumento de la población regional del 1,86\%, pasando de 1.628.005 habitantes en 1981 a 1.658 .445 en 1991; «se está experimentando en la actualidad la transición de un crecimiento anual de cero negativo a cero positivo, lo que equivaldría a poder afirmar que, por primera vez desde 1950, la región, en lugar de perder población comienza a recuperarse»" ${ }^{2}$.

El crecimiento intercensal en las provincias castellano-manchegas es análogo en todas ellas y va en aumento desde 1900 a 1950 . Es a partir de este año cuando se encuentran las diferencias. Toledo y Albacete ven disminuir su población desde 1950, rompiéndose esta tendencia en el censo de 1991, en el que se observa un ligero crecimiento. En Cuenca y Guadalajara también disminuye la población desde el censo de 1950, sin embargo no se contempla ningún aumento en 1991. En Ciudad Real el descenso de la población se inicia a partir del censo de 1970 y hay un pequeño aumento en el censo de 1991.

\subsection{Los movimientos migratorios y el crecimiento vegetativo.}

Los movimientos migratorios y el crecimiento vegetativo han sido los dos factores demográficos más importantes en este proceso evolutivo. La región de

1. Molina, M. La población de Castilla-La Mancha. II Reunión de estudios regionales de CastillaLa Mancha. El espacio rural de Castilla-La Mancha. Tomo II. Ciudad Real: Diputación de Ciudad Real, 1986. Biblioteca de Autores Manchegos, p. 89.

2. GonZÁlez, E.; PILLER, F. Geografía fisica, humana y económica de Castilla-La Mancha. Ciudad Real: Diputación de Ciudad Real, 1986. Biblioteca de Autores Manchegos, p. 87. 
Castilla-La Mancha, desde principios del siglo hasta la actualidad, ha sufrido pérdidas de población, si bien es a partir de los años cincuenta cuando apareció el boom de la emigración, lo que conllevó a una gran despoblación y a un envejecimiento de la población, sobre todo en el medio rural. Desde 1981 a la actualidad se observa un descenso de la emigración y un ligero aumento de la inmigración: en 1993 el número de salidas fue de 28.642 personas y el número de entradas de 32.471 , lo que supone un saldo migratorio favorable de 3.829 personas para ese año.

En las provincias se da, en líneas generales, el mismo comportamiento que en la región. Hasta 1950 existe una tendencia de saldos migratorios negativos, a excepción de Ciudad Real y Albacete, que reciben una ligera inmigración; a partir de 1950 hasta 1980 las pérdidas aumentan en todas y cada una de las provincias, y es desde 1981 cuando se puede contemplar un pequeño aumento de población inmigrante en Guadalajara, Toledo y Cuenca. No obstante, «esas llegadas actuales son tan poco importantes que no tienen apenas incidencia en la evolución demográfica regional, si bien ha frenado la despoblación masiva de años anteriores» ${ }^{3}$. En el año 1993 el saldo migratorio ha sido positivo en Toledo $(+2.587)$, Guadalajara $(+1,719)$ y Albacete $(+191)$, mientras que en Cuenca $(-455)$ y Ciudad Real $(-213)$ ha sido negativo.

Con relación al crecimiento vegetativo de Castilla-La Mancha, hay que seńalar que a lo largo del siglo XX se ha daclo un incremento natural positivo, y en líneas generales se ha producido un retroceso progresivo de la natalidad y de la mortalidad. "Castilla-La Mancha ha culminado su modelo de transición demográfica por envejecimiento de la población, si bien éste no obedece a una elevación sustancial del nivel de vida, que progresivamente ha ido generando una natalidad baja, sino a la emigración de gente joven ${ }^{4}$. En las diferentes provincias la evolución del crecimiento natural es parecida a la de la región, aunque hay que resaltar que en Cuenca y Guadalajara las tasas de natalidad son las más bajas, las tasas de mortalidad las más altas y su crecimiento vegetativo es, en los últimos años, negativo.

\subsection{La esperanza de vida al nacer}

En cuanto a la esperanza de vida al nacer las ganancias han sido muy importantes, y son debidas principalmente a la radical reducción de la mortalidad infantil y a la disminución de la mortalidad en edades avanzadas. Entre 1971 y 1991 la esperanza media de vida ha pasado de 69,85 años a 75,29 para los varones, y de 74,06 a 80,27 para las mujeres.

3. AA.VV. «Caracteres geodemográficos». Papeles de Economía Española. Madrid: CECA, 1987, p. 77.

4. AA.V. "Caracteres geodemográficos". Papeles de Economia Española. Madrid: CECA, 1987, p. 77. 


\subsection{Las proyecciones de la población}

Con respecto a la evolución de la población en los próximos años, según previsiones del Instituto de Demografía ${ }^{5}$, la Comunidad de Castilla-La Mancha del año 1991 al 2006, si se cumplen las hipótesis de la proyección, aumentará su población moderadamente. Se parte de una población de 1.658 .377 en 1991 para alcanzar 1.672.473 de habitantes en el año 2006. En comparación con las otras comunidades autónomas, en once de ellas, más Ceuta y Melilla, la población proyectada aumenta (Cataluña, Madrid, las comunidades del este $\mathrm{y}$ del sur, $\mathrm{y}$ las insulares) y disminuye en las seis restantes.

Asimismo, el Instituto de Demografía ha medido el dinamismo demográfico de las comunidades autónomas calculando un índice que relaciona la proporción del crecimiento total de cada región con la proporción que su población representa, al inicio del periodo, con el total de la población española. Cuando el valor de este índice es cero el porcentaje del crecimiento coincide con el porcentaje de la población (es decir, la región conserva su situación en el conjunto del Estado), los valores negativos muestran un dinamismo demográfico inferior a la media. Según este índice de dinamismo demográfico, la comunidad de Castilla-La Mancha tiene un valor negativo $(-0,80)$ en el periodo 19912006, al igual que las comunidades de Aragón, Asturias, Cantabria, Castilla-León, Cataluña, Extremadura, Galicia, Navarra, País Vasco y La Rioja.

\subsection{La estructura por edades}

La estructura por edades de la comunidad de Castilla-La Mancha, en el año 1991, es similar a la del Estado español en el grupo de población de 0-14 años, siendo el $19,50 \%$ de la población en la primera y el 19,36\% en el segundo. En los otros dos grupos de edad, si en España la población de 15 a 64 años es mayor (66,5\% frente a 63,7\%), en el grupo de más de 65 años es menor (13,8\% frente a 16,3\%), lo que nos indica que la población manchega está más envejecida en comparación con la población total española. La diversidad que existe en la estructura por edades entre las distintas provincias que comprenden la Comunidad se da en los grupos de menos de 14 años y más de 65 años. La población más joven se encuentra en la provincia de Albacete $(21,2 \%)$, seguida de Ciudad Real $(19,9 \%)$ y Toledo $(19,7 \%)$, en tanto que Cuenca y Guadalajara tienen ambas el menor porcentaje de población de menos de 14 años $(17,2 \%)$. Esto se corresponde, a su vez, con el grupo de población de más de 65 años, pues son estas dos últimas provincias las que tienen el mayor porcentaje de población de más edad (19,3\% y 19\% respectivamente), seguidas de Toledo (16,1\%) y Ciudad Real (16,3\%); siendo Albacete la provincia que tiene menos porcentaje de población envejecida $(13,8 \%)$. $\mathrm{Y}$, en cuanto al grupo de 15 a 64 años, es muy parecido en todas la provincias, estando en torno al 63\%, a excepción de Albacete, que es el 64,6\%. 
Si atendemos a la diferenciación entre sexos el mayor porcentaje de varones se encuentra en todas las provincias en los grupos de 0 a 14 años y de 15 a 64 años, situándose las mujeres en el. grupo de más de 65 años. Esta misma situación se produce en el Estado español.

En las proyecciones de población efectuadas por el Instituto de Demografía se puede advertir que en la comunidad de Castilla-La Mancha el grupo de 0 a 19 ańos disminuye, pasa del $27,71 \%$ en 1991 al $23,58 \%$ en el 2006 , mientras que en el grupo de 20 a 59 años aumenta (del $4: 9,84 \%$ al 52,89\%), al igual que el grupo de más de 60 años ( $d e l$ 22,46\% al 23,53\%). Esta situación es debida a que la tasa de crecimiento anual pasará del 0,88 por mil (en 1991) al 0,78 por mil (en el 2006). No obstante el Indice Sintético de Fecundidad, si en 1991 es de 1,57 hijos por mujer, en el año 2005 se prevé que sea del 1,73 hijos por mujer, lo que supone una disminución del proceso de envejecimiento de esta comunidad.

Finalmente, resaltar que la esperanza media de vida al nacer seguirá aumentando, según las previsiones demográficas apuntadas anteriormente, y para el año 2006 será de 76,85 años para los varones y de 82,26 para las mujeres.

\section{Hogares y viviendas}

En la región de Castilla-La Mancha hay 509.877 hogares, y su tamaño medio es de 3,22 personas por hogar, muy similar a los del Estado español que es de 3,28 . En el $38,1 \%$ de éstos reside algín miembro menor de 16 años y en el $38,9 \%$ lo hace algún miembro de 65 o más años, mientras que a nivel estatal estas cifras son del $40,3 \%$ en el primer grupo y del $34,1 \%$ en el segundo grupo. Según el estado civil de los sujetos la mayoría de los hogares cuenta con algún miembro casado $(77,9 \%)$ o con algún miembro soltero $(68,4 \%)$, los viudos viven en el $21 \%$ de los hogares, sólo con miembros solteros hay un 5,6\%, y los divorciados sólo se encuentran en el $0,3 \%$ de los hogares.

Si nos atenemos a la relación con el parentesco, en la inmensa mayoría de los hogares castellano-manchegos $(95,3 \%)$ el sujeto vive con sus familiares: el $56,9 \%$ vive con el cónyuge, el $45,6 \%$ con los hijos, el $30 \%$ con el padre y la madre o con los hermanos, y sólo el $3 \%$ con los abuelos, cifras muy parecidas a la media nacional. Los hogares unipersonales representan el 4,6\% del total.

En cuanto a las viviendas, en los últimos años el $71 \%$ de la población manchega no ha efectuado ningún cambio de vivienda, sí lo han hecho el 18\% dentro del propio municipio y el $11 \%$ cambiando a otro municipio. De los que han cambiado el $52,7 \%$ vivía en ella hacía más de 10 ańos, y el 28,2\% menos de 5 años. Los motivos del cambio han estado asociados a un cambio de población de residencia en un $30 \%$, y del resto el $24,2 \%$ lo hizo por circunstancias personales (jubilación, nuevo empleo, enfermedad, matrimonio), el $20,4 \%$ por adquisición de otra vivienda y el $19,1 \%$ por otras razones (una vivienda mejor, una vivienda más grande, una más económica, etc.).

Respecto a la titularidad de la vivienda, en el $60 \%$ de ellas su propietario es el sujeto o su cónyuge, el $34,7 \%$ es de los padres o suegros, el 1,9\% de los hijos o yernos/nueras, y a otro pariente corresponde el $2,9 \%$. Y, en relación con las 
viviendas secundarias, el $91,2 \%$ de la población no dispone de ellas, sólo el $8,8 \%$ tiene una y el $0,8 \%$ dispone de dos o más viviendas. En este tipo de viviendas, se encuentra el $57,1 \%$ de ellas en el mismo municipio que la vivienda principal, el $24,6 \%$ en otro municipio de la provincia, el $16,5 \%$ en otra comunidad autónoma y el $1,8 \%$ en otras provincias de la propia comunidad. El uso que se les da en el $58,2 \%$ de los casos es para utilizarlas los fines de semana o algunos días tan sólo, el 25,8\% en temporadas cortas (hasta 3 meses), y el 14,7\% en temporadas largas (más de 3 meses).

\section{Movimientos migratorios}

La mayoría de los castellano-manchegos $(67,4 \%)$ sigue residiendo en su lugar de nacimiento. El 72,3\% lleva viviendo en su residencia actual entre el $80 \%$ y el $100 \%$ de su vida y el $7,8 \%$ lleva menos del $20 \%$. Han cambiado de lugar de residencia dentro de la propia provincia un $13,7 \%$, un $2,1 \%$ dentro de la comunidad, el $15,7 \%$ a otras comunidades, y sólo el $1,2 \%$ a otro país. La mayoría de los que han variado de residencia $(44,7 \%)$ tenían entre 15 y 30 años, y los motivos han sido laborales en un $39,8 \%$, familiares en un $13,9 \%$, y por otros motivos ocupacionales en un $4,5 \%$.

\section{Formación}

El $85 \%$ de la población manchega ha cursado algunos estudios, mientras que el $15 \%$ restante no ha realizado ningún tipo de estudios. Este último porcentaje es muy elevado si lo comparamos con la media nacional que es del $7,8 \%$, y es a la vez el porcentaje más alto de todas las regiones españolas, seguido de Ceuta y Melilla que tienen un 13,5\% y Andalucía con un 12,9\%. De las personas que no han hecho estudios el $7,5 \%$ es analfabeto, cifra que sólo es superada por Ceuta y Melilla (8,7\%) y Extremadura (7,7\%). Este índice de analfabetismo se concentra en las personas de más de 50 años y más en las mujeres que en los hombres.

Iniciaron sus estudios de primer grado el $84,7 \%$; el segundo grado ( $1 \mathrm{r}$ nivel) el $43,2 \%$; el segundo grado ( $2^{\circ}$ nivel inferior), el $70 \%$; el segundo grado ( $2^{\circ}$ nivel superior), el 19\%; el tercer grado ( $1 \mathrm{r}$ nivel), el $4,6 \%$; el tercer grado ( $2^{\circ}$ nivel), el $3,7 \%$, y el tercer grado (nivel superior), el $0,2 \%$; los finalizaron el $65,6 \%$, el $32,1 \%$, el $4,3 \%$, el $12,5 \%$, el $3,4 \%$, el $1,8 \%$ y el $0,2 \%$ respectivamente. De estos datos hay que destacar que esta comunidad es la región española donde menos población comienza estudios y donde menos los finaliza, únicamente en Extremadura, Ceuta y Melilla se obtienen cifras parecidas.

De los que dejaron de estudiar el $33,4 \%$ es porque habían concluido lo que querían hacer, el 19,8\% porque no les gustaba estudiar o no aprobaban y el $46 \%$ los abandonó por circunstancias personales. Entre estas circunstancias destacan: porque su ocupación se lo impedía $(33,5 \%)$, porque estaba ocupado en una actividad económica $(27,1 \%)$ y porque carecía de recursos $(13 \%)$. Cifras estas muy similares a la media nacional. 


\section{Población activa e inactiva}

La población activa de la comunidad castellano-manchega es inferior a la media nacional, puesto que a nivel estatal el $49,21 \%$ de la población de 16 y más años es considerada activa, mientras que en la región esta población es del 43,36\%. En las provincias, Albacete es la que más se aproxima a la media estatal con un $48,71 \%$ de población activa y las más lejanas son Cuenca con un $39,11 \%$ y Guadalajara con un 40,85. Como consecuencia, el mayor número de inactivos se concentra en Cuenca y Guadalajara, alcanzando el $55 \%$ en Ciudad Real y Toledo, y el número menor es en Albacete, aunque también la cifra es elevada, pues supone un poco más de la mitad de la población de 16 y más años $(50,81 \%)$.

De la población activa los ocupados en Castilla-La Mancha son el 81,27\%, cifra superior a la del total de España, que es del 77,27\%. En la distribución por provincias podemos observar como Guadalajara y Cuenca tienen el mayor porcentaje de población ocupada, $87,86 \%$ y $82,16 \%$ respectivamente, seguidas de Toledo $(81,47 \%)$ y Ciudad Real $(79,14 \%)$, y la provincia que tiene menos ocupados es Albacete, con un $75,74 \%$. En cuanto a la población que se encuentra en situación de paro, el porcentaje de la región se encuentra cuatro puntos por debajo del total nacional, sin embargo en la provincia donde más paro se da es en la de Albacete (24,26\%) y donde menos es en Guadalajara (12,14\%), seguida de Cuenca $(17,84 \%)$ y Toledo $(18,53 \%)$, y en Ciudad Real el porcentaje de población parada es del $20,86 \%$, cifra tambien menor a la alcanzada en el Estado español.

En relación con el sexo, los varones activos ocupan un porcentaje menor que el resto de la nación, en la región son el 59,38\% y en aquélla son el 62,96\%. Este porcentaje es mayor cuando se refiere a las mujeres, pues se da una diferencia de casi ocho puntos a favor de la media estatal, $28,13 \%$ para las castellano-manchegas y $36,41 \%$ para el total de España. Y cuando comparamos las cifras de población activa ocupada, la diferencia es mayor entre los varones castellano-manchegos y los del total nacional, que entre las mujeres de la región y las de España; pues entre los primeros se da una diferencia de casi cuatro puntos ( $86,38 \%$ y $82,05 \%$ respectivamente) y en relación con las mujeres se dan cifras similares $(71,07 \%$ y $69,78 \%)$. A nivel provincial los porcentajes son muy parecidos en cada una de las provincias. Excepción hecha de los varones de Cuenca y Guadalajara, pues cerca de la mitad de ellos son inactivos y de los activos de Guadalajara están ocupados más del $92 \%$.

Consideramos importante resaltar otra cuestión respecto a la población activa de los ocupados según la situación profesional. De los datos que hemos obtenido del censo de 1991 al comparar la región con la nación destaca principalmente que los porcentajes de ocupados son casi iguales en los grupos de "empresarios con asalariados", "empresarios sin asalariados", "miembro de cooperativa» y "ayuda familiar». Sin embargo, sí que existe diferencia en cuanto a los "asalariados fijos" y a los «asalariados eventuales», pues si en España el primer grupo lo componen el $49,44 \%$ de los ocupados y el segundo grupo el 
$24,80 \%$, en la región el porcentaje es del $40,87 \%$ y del $27,52 \%$ respectivamente. Estas cifras son muy semejantes en cada una de las provincias, si bien hay que mencionar un dato que sobresale sobre los demás: es el número de empresarios sin asalariados que hay en Cuenca, pues alcanzan un $25,60 \%$ de la población ocupada en esa provincia.

\section{Conclusiones}

Como conclusiones finales podemos señalar que la región de Castilla-La Mancha es una comunidad con una gran superficie apenas poblada $\left(78 \mathrm{~h} / \mathrm{km}^{2}\right)$, desequilibrada territorialmente al agruparse la población en los núcleos urbanos y dominada por pequeños municipios con una población en regresión y con tendencia a la desaparición.

En su conjunto la población regional en los últimos años va aumentando ligeramente. Sin embargo esto no sucede así en la provincia de Cuenca, en la que la población disminuye desde los años sesenta. Esta evolución de crecimiento, según diversas proyecciones de población, seguirá en los años venideros, aunque será un aumento moderado y con un dinamismo demográfico inferior a la media nacional.

Los movimientos migratorios y el crecimiento vegetativo han sido los factores demográficos que más han condicionado la región casteliano-manchega. La emigración ha traído consigo una gran despoblación y un envejecimiento de la población, incidiendo mucho más en el mundo rural; no obstante, desde 1981 ha descendido la emigración y se ha producido un pequeño aumento en la inmigración. $Y$ en relación con el crecimiento vegetativo se ha dado, a lo largo del presente siglo, un retroceso progresivo de las tasas de natalidad y mortalidad, lo que ha supuesto un incremento natural positivo para la población regional, aunque hay que destacar que las provincias de Cuenca y Guadalajara tienen las tasas de natalidad más bajas y las tasas de mortalidad más altas, y que su crecimiento vegetativo es actualmente negativo.

En cuanto a la estructura por edades, el grupo de 0-14 años es un colectivo de 324.182 personas (19,5\%), el de 15-64 ańos lo componen 1.057.154 personas y en el de más de 65 años hay 270.862 individuos. Al comparar estas cifras con las estatales podemos ver como las que corresponden al primer grupo son parecidas y como la población manchega está más envejecida que la población total española. La población más joven es la de la provincia de Albacete, y se encuentra a cuatro puntos de diferencia la juventud de Cuenca y Guadalajara, que son las que tienen, a su vez, la población de más edad, siendo el grupo de 15-64 años similar en todas las provincias.

Se prevé que estos grupos de edad variarán en los próximos años, disminuyendo el grupo de 0-19 años, y aumentando el de 20-59 años y el de más de 60 años, aunque también se contempla en las proyecciones de población que el índice sintético de fecundidad actual aumente en el año 2005, consiguiéndose así una disminución del proceso de envejecimiento. 
De los hogares castellano-manchegos destaca que: en la gran mayoría de ellos el sujeto vive con sus familiares, se cambia muy poco de vivienda, la mayoría es propietario de su vivienda y no se suele tener una vivienda secundaria.

Respecto al nivel de instrucción de la población manchega, resaltar que el $85 \%$ ha cursado algunos estudios y el resto no ha efectuado ningún tipo de ellos, porcentaje este último muy elevado al compararlo con la media nacional $(7,8 \%)$, siendo el más alto de todas las regiones españolas. Nos encontramos con un $7,5 \%$ de analfabetos, que se concentran en las personas mayores y más en las mujeres. Es la región española donde menos población inicia estudios y donde menos los terminan.

Por último, en relación con la población activa e inactiva de la región hemos de considerar que la población activa es inferior a la media nacional, el mayor número de activos se encuentra en la provincia de Albacete y el mayor número de inactivos en Cuenca y Guadalajara. Sin embargo, de la población activa el porcentaje de ocupados es mayor en Castilla-La Mancha que en España, y donde más activos ocupados se da es en las provincias de Guadalajara y Cuenca. La situación de paro es más baja en la región que en el resto del país, la provincia de más paro es Albacete y la de menos es Guadalajara. Y, en cuanto al sexo, son los varones los que ocupan el mayor porcentaje de población activa.

\section{Bibliografía}

AA.VV. (1993). El impacto de las políticas sociales: educación, salud, vivienda. I Simposio sobre igualdad y distribución de la renta y la riqueza. Vol. VIII. Madrid: Fundación Argentaria.

AA.V. (1995). España 1994. Una interpretación de su realidad social. Centro de estudios del cambio social. Madrid: Fundación Encuentro.

AA.VV. (1994). VInforme sociológico sobre la situación social en España. Sociedad para todos en el año 2000. Madrid: Fundación FOESSA.

BLEDA GARCíA, J.M. (1996). Sociedad y vejez. Las políticas sociales para la vejez: percepción, tendencias y lineas de desarrollo. El caso de Castilla-La Mancha. Tesis doctoral. Universidad de Murcia.

INE (1992). Censo de Población y Viviendas 1991. Madrid.

INE (1993). Encuesta Sociodemográfica 1991. Volúmenes 1, 2 y 3. Madrid.

INE (1995). Encuesta de Población Activa. Principales resultados. Tercer trimestre. Madrid. INSTITUTO DE DEMOGRAFIA (CSIC) (1994). Proyección de la población española. España 1991-2006. Comunidades Autónomas 1991-2006. Provincias 1991-2006. Madrid.

JunTA DE COMUnidades de CASTILla-La MANCHA (1994). Guía de recursos culturales de Castilla-La Mancha. Toledo.

UNÁ JuÁreZ, O. «Elecciones políticas y conciencia regional en Castilla-La Mancha». Enciclopedia de Castilla-La Mancha (en prensa).

UNA, O.; BLEDA, J.M. (1996). "Las fuerzas políticas y sociales». Situación. Bilbao: BBV (en prensa).

UNAA, O.; BLEDA, J.M. (1996). «La Universidad de Castilla-La Mancha, motor de cambios sociales, culturales y económicos». Sociedad y Utopia. Madrid (en prensa). 